\title{
Agile Shopping of Grocery Items in a Mart
}

\author{
Rajalakshmi N ${ }^{1}$, B.E. (Scholar), Ramya Namasivayam², B.E. (Scholar), \\ Renukadevi SR ${ }^{3}$, B.E. (Scholar), Jananee $\mathrm{V}^{4}$, \\ Assistant Professor, Department Of Computer Science and Engineering Rajalakshmi Engineering College \\ Thandalam, Chennai
}

\begin{abstract}
The concern of many organisations is to minimize the total time consumption taken for purchasing and billing. Through the analysis of customer behaviour, accurate profiles are being generated by specifying needs and interest and allowing organisation to give customers what they want it, when they want, leading to a better customer satisfaction thereby keeping them to come back for more. To improve the business performance of an organisation we need to automate the right offers to the right person at the right time. Our project provides the unique id for each registered customer. Based on registration the individual can start their shopping. After purchasing the product, they can scan the products using mobile application and the concerned bill for the particular customer will be intimated to the retailer, the number of products purchased will be checked and cash is paid. It reviews the status of products availability and it notifies to the retailer if it reaches concerned limit. This project provides the brief view of business liabilities to the retailers using apriori algorithm. This project will help the customer to purchase the products through online. Online shopping will also be useful for analysing the customer's previous shopping history.
\end{abstract}

Keywords: Customers Behaviour, Automate, Mobile Application, Apriori Algorithm

\section{INTRODUCTION}

The main objective of this application is to reduce the time spent in the queue of the customer for long time and to help the adult people to making the billing process easier for them. The development of this application will reduce the employees work in the supermarket. In the existing system, the users have to stand in the queue to generate the manual bill generated in the super markets. Usually, in the super markets the user purchase the products after the purchased products are sent to the billing process [1]. There will be $\mathrm{N}$ number of users will be waiting in the billing process. At that time the user have to wait in the queue for the long time which is time consuming. In the proposed system, the user needs not to stand in the queue for a long time. They just have to scan the bar code of the product. After scanning of the product the product details and the user details are sent to the billing process. After that the bill is generated for the particular user. The bill is generated automatically based on the product list. The scope of this system is to provide the app through which we can easily generate the bills in the super markets and also to reduce the customer waiting time spending in the manual bill generation and is used to develop an app which is useful for the adults to avoid the waiting in the queue for the bill generation in the super markets. A bar code system enables a strict sales and inventory control that as previously unattainable. In the mart, a product which is sold is identified by its bar code at the checkout [2]. This information is then sent to the cash register as well as to the inventory and administration. The customer gets an itemised receipt of his purchases and the stores is able to keep a close watch on goods that are sold, going out of stock (inventory control) as well as provide information on consumer behaviour (administration and statistics). The store can then order new stocks automatically depending on the level of automation and the software used - or generates a list for the sales staff and reports for the administration. The whole bar code system needs software to run. Some systems have their own embedded software but bar code scanners and printers can also be hooked up to a personal computer running Windows, Apple OS or Linux software. Verification of the bar code should be done after the printing of the label as well after scanning it. A properly verified bar code label means that it can be scanned (read) by all suitable scanners, not just the one you are using. Online Shopping is implemented with XAMPP software is used to connect PHP with MySQL Database and Apache Server is used to connect web pages [3]. With the rapid expansion of e-commerce, more and more products are sold on the Web, and more and more people are also buying products online. In order to enhance customer satisfaction and shopping experience, it has become a common practice for online merchants to enable their customers to reviewer to express opinions on the products that they have purchased. With more and more common users becoming comfortable with the Web, an increasing number of people are writing reviews. As a result the number of reviews that a product receives 
grows rapidly. Some popular products can get hundreds of reviews at some large merchant sites. Furthermore, many reviews are long and have only a few sentences containing opinions on the product. This makes it

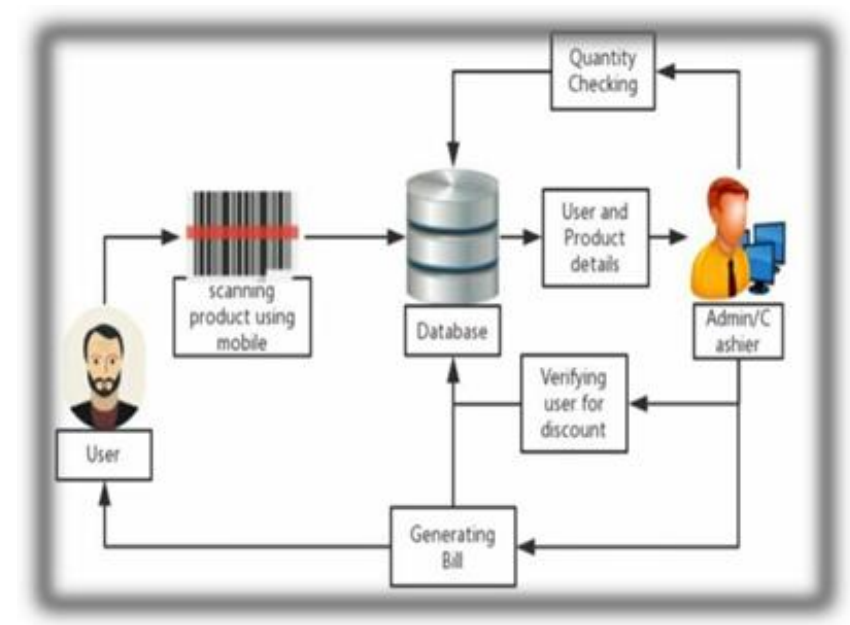

Fig: Architecture Diagram

hard for a potential customer to read them to make an informed decision on whether to purchase the product.

\section{FEASIBILITY STUDY}

The feasibility of the project is analyzed in this phase and business proposal is put forth with a very general plan for the project and some cost estimates. During system analysis the feasibility study of the proposed system is to be carried out [4]. This is to ensure that the proposed system is not a burden to the company. For feasibility analysis, some understanding of the major requirements for the system is essential. Three key considerations involved in the feasibility analysis are

\subsection{ECONOMICALFEASIBILITY}

This study is carried out to check the economic impact that the system will have on the organization. The amount of fund that the company can pour into the research and development of the system is limited. The expenditures must be justified [10]. Thus the developed system as well within the budget and this was achieved because most freely available. Only the customized products of the technologies used are had to be purchased.

\subsection{TECHNICALFEASIBILITY}

This study is carried out to check the technical feasibility, that is, the technical requirements of the system. Any system developed must not have a high demand on the available technical resources [5]. This will lead to high demands on the available technical resources. This will lead to high demands being placed on the client. The developed system must have a modest requirement, as only minimal or null changes are required for implementing this system.

\subsection{SOCIAL FEASIBILITY}

The aspect of study is to check the level of acceptance of the system by the user. This includes the process of training the user to use the system efficiently. The user must not feel threatened by the system, instead must accept it as a necessity. The level of acceptance by the users solely depends on the methods that are employed to educate the user about the system and to make him familiar with it. His level of confidence must be raised so that he is also able to make some constructive criticism, which is welcomed, as he is the final user of the system.

\subsection{MODULE DESCRIPTION}

\subsubsection{USER LOGIN}

In this we will have authentications for all the users, admin. In this product scanning will be based on user Id. If Id is not there they can't access this system. Only with this Id only they are going to give products. 2.4.2 SCANNING PRODUCTS

User will scan the Bar Code and it will be scored through mobile phone and those details will be redirected for billing. In that it will give you the details regarding user details and also product details 


\subsubsection{BILL GENERATION}

This module will display all the detail of user and product to that particular cashier and then with that they will generate the bill to that particular user with complete details of billing.

\subsubsection{VERIFYING USER DETAIL FOR DISCOUNT}

In this module whenever a customer enters that system will add visiting time in database. Finally when they visit 5 or more than 5 times then we will give them a discount on their purchase to support regular customers.

\subsubsection{QUANTITY MONITORING}

In this module we will monitor all the products in daily basis and in this they will take access as number of products sold out, number of products needed for next day and stock left. All this will be monitoring by the admin on daily basis.

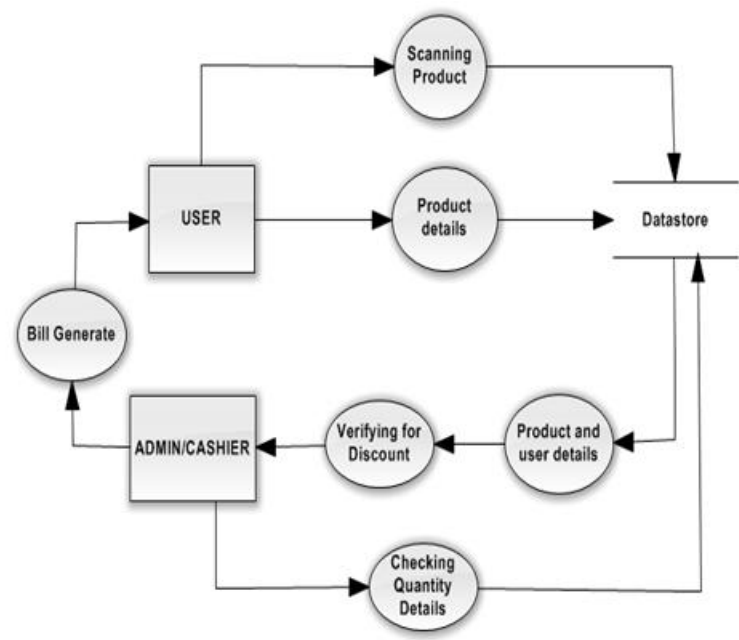

Fig: Process Flow Representation

\section{BACKGROUND STUDY}

Merchants selling products on the Web often ask their customers to review the products that they have purchased and the associated services. As e-commerce is becoming more and more popular, the number of customer reviews that a product receives grows rapidly. For a popular product, the number of reviews can be in hundreds or even thousands. This makes it difficult for a potential customer to read them to make an informed decision on whether to purchase the product. It also makes it difficult for the manufacturer of the product to keep track and to manage customer opinions [6]. For the manufacturer, there are additional difficulties because many merchant sites may sell the same product and the manufacturer normally produces many kinds of products. [7] In this paper, we aim to mine and to summarize all the customer reviews of a product so that it helps in giving a good opinion about the product and that results in better shopping.

Data mining, in the recent times has gained a lot of popularity due to the availability of huge sets of data, which grows day by day. The need to transform this data into useful information has lead to the development of various data mining algorithms. The Apriori algorithm, known to be one of the best algorithms for finding frequent item sets in large transactional databases, is the subject of research in this paper. In Retail Industry, which accumulates a huge amount of sales data on a daily basis, there is a constant need for analyzing data to determine frequently purchased items by a customer, over a period of time. An instance for usefulness of this information can be as follows. This information can be used to generate a shopping cart of items for the customer who is deliverable on demand. The paper discusses also discusses the advantages and disadvantages of Apriori Algorithm [8]. It also explains the implementation of Apriori Algorithm in various domains. Specifically in the retail industry; frequent patterns are mined from previous transaction records of customers. The retailers can then use such information to analyze the frequent items bought by the customer, to understand their interests, to satisfy their demands and above all to predict their needs. As seen in the past, Retail Stores have attained success by emphasizing on understanding customer buying-habits from these sets of data.

\section{Analysis Report}

The purchase history of the products for a particular id is analysed and the analysis report is generated. This report helps in finding the profit analysis for each items in the grocery store. 


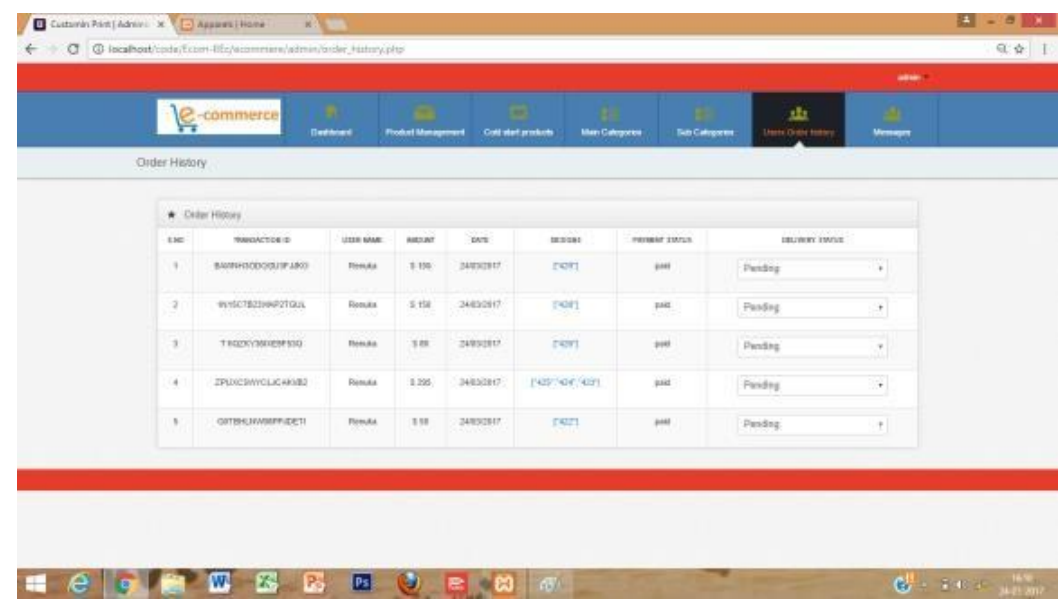

\section{CONCLUSION}

In this paper we described how Apriori algorithm is used for discovering locally frequent patterns from retail data sources. Various data mining techniques were used earlier for pattern analysis. However, for finding locally frequent items, Apriori is most suitable especially for transactional databases. This has lead to various improvisations of the core approach. However this technique is useful for only small datasets. For larger datasets, generation of candidate sets and finding frequently occurring items is time consuming and complex [9]. As the size of data increases, the algorithm takes more number of data scans and eventually greater number of iterations for deriving the strong association rules. In this application we learned the architecture diagram and the work flow of the project. Also we learned that how the product details and the user details are obtained from the bar code of the product, how the bills are generated in the super markets by scanning the bar code of the product. Through this application it is ensured that this is the useful application for the users to minimize the time spent to generate the bills in the super markets. [10] Hence the system proposes the automation for automatic barcode scanning with billing system. This will avoid the waiting time for billing and more over there is no need to use more workers in billing section. Our system takes less time for the scanning compared to handheld method. It also describes how Apriori algorithm is used for discovering frequent patterns from retail data sources. The product to be developed is easy to use, low-cost and does not need any special training.

\section{REFERENCES}

[1]. Agrawal, R. \& Srikant, R. 1994. Fast algorithm for mining association rules. VLDB'94, 1994.

[2]. 2] R. Sumithra, Using Distributed Apriori Association Rule and Classical Apriori Mining Algorithms for Grid Based Knowledge Discovery, International Conference on Computing Communication and Networking Technologies, 2010,

[3]. Andrew Ainslie, Peter E. Rossi. (1998). Similarities in Choice Behavior Across Product Categories. Marketing Science, Vol.17, No.2,91-106.

[4]. Prof. Kavitha Venkatachari Issac Davanbu Chandrasekaran IBS Business School, Powai, Mumbai, India

[5]. W. J. Pei, Mining Association Rules Based on Apriori Algorithm and Application”, International Forum on Computer ScienceTechnology and Applications, 2009

[6]. David R. Bell and James M. Lattin. (2008). Shopping Behavior and Consumer Preference for Store Price Format: Why "Large Basket”. Marketing Science, Vol. 17, No. 1, 66- 88.

[7]. KatrinDippold, Harald Hruschka. (2010). Variable Selectio for Market Basket Analysis. University of Regensburg Working Papers in Business, Economics and Management Information Systems.

[8]. Sanjeev Rao and Priyanka Gupta, "Implementing Improved Algorithm Over APRIORI Data Mining Association Rule Algorithm," IJCST Vol. 3 .

[9]. Jiawei Han and Micheline Kamber, "Data Mining Concepts and Techniques "Second Edition. Morgan Kaufmann Publisher, 2006, Pp.123-134.

[10]. Agrawal R and Imielinski T and Swami A N Mining association rules between sets of items in large databases; In Proceedings ACM SIGMOD International Conference on Management of Data Vol 22; No 2; of SIGMOD Record; Washington; pp 207$216 ; 1993$. 